\title{
Knockdown of RWD domain containing 3 inhibits the malignant phenotypes of glioblastoma cells via inhibition of phosphoinositide 3-kinase/protein kinase B signaling
}

\author{
XIAOFENG CHEN, WEIPING KUANG, HONGXING HUANG, BO LI, YONG ZHU, BIN ZHOU and LIN YAN \\ Department of Neurosurgery, Brain Hospital of Hunan Province, Changsha, Hunan 410007, P.R. China
}

Received July 9, 2017; Accepted October 13, 2017

DOI: $10.3892 /$ etm.2018.6135

\begin{abstract}
Glioblastoma is the most common and malignant primary brain tumor. RWD domain containing 3 (RWDD3) has been previously reported to serve a promoting role in pituitary tumors. However, the exact role of RWDD3 in glioblastoma remains unclear. Therefore, the present study aimed to investigate the expression levels of RWDD3 in human glioblastoma tissues and cell lines, as well as to examine the regulatory mechanism of RWDD3 underlying glioblastoma growth and metastasis. The results revealed that RWDD3 was significantly upregulated in glioblastoma tissues compared with normal brain tissues, while high expression of RWDD3 was associated with a shorter survival time of glioblastoma patients. The expression levels of RWDD3 were also higher in the glioblastoma cell lines compared with the normal human astrocyte cell line. Subsequent to knockdown of RWDD3, the proliferation of glioblastoma U87 and U251 cells was significantly decreased, possibly due to the cell cycle arrest at G1 phase, as well as the increased cell apoptosis. Furthermore, downregulation of RWDD3 also suppressed U87 and U251 cell invasion by inhibiting the expression levels of matrix metalloproteinase 2 (MMP2) and MMP9. Molecular mechanism investigation demonstrated that knockdown of RWDD3 significantly downregulated the activity of the phosphoinositide 3-kinase/protein kinase B (PI3K/AKT) signaling pathway. Activation of PI3K/AKT signaling prevented the suppressive effects of RWDD3 downregulation on glioblastoma cell proliferation and migration, concurrent with increased protein levels of MMP2 and MMP9. In conclusion, the current study demonstrated for the first time that inhibition of RWDD3 expression inhibited glioblastoma progression, at least partly, via suppressing the PI3K/AKT signaling activity,
\end{abstract}

Correspondence to: Professor Weiping Kuang, Department of Neurosurgery, Brain Hospital of Hunan Province, 427 Furong Middle Road, Changsha, Hunan 410007, P.R. China

E-mail: kuangweiping2017@163.com

Key words: glioblastoma, RWD domain containing 3, oncogene, tumor growth and thus RWDD3 may be a novel potential therapeutic target for glioblastoma.

\section{Introduction}

Glioblastoma is the most aggressive cancer of the brain (1). Despite advances in recent decades on the treatment of glioblastoma, including surgical resection combined with radiotherapy and chemotherapy, the survival time of glioblastoma patients remains unsatisfactory, with a median survival period of $\leq 12$ months (1-3). Increasing evidence has demonstrated that the poor prognosis of glioblastoma patients is largely attributed to the intrinsic molecular changes, and several oncogenes and tumor suppressors have been identified in the process of glioblastoma progression, certain of which may be potential therapeutic targets (2-4).

RWD domain containing 3 (RWDD3), also known as RWD domain-containing sumoylation enhancer), was previously reported to be highly expressed in the cerebellum, pituitary, heart, kidney, liver, pancreas, adrenal gland and prostate (5). RWDD3 has been demonstrated to enhance glucocorticoid receptor sumoylation and transcriptional activity (6,7). It interacts with the small ubiquitin-like modifier (SUMO) conjugating-enzyme Ubc9 and consequently increases Ubc9 thioester formation, thus favoring sumoylation of specific targets (5). During cellular stress, such as that induced by hypoxia and heat shock, RWDD3 was observed to inhibit the nuclear factor- $\kappa \mathrm{B}$ signaling through increasing the $\mathrm{I} \kappa \mathrm{B}$ levels, and to stabilize hypoxia-inducible factor (HIF)- $1 \alpha$ via suppressing HIF-1 and $-2 \alpha$ ubiquitination and degradation $(5,8)$. Recently, Wu et al (9) reported that loss of RWDD3 is crucial for pancreatic neuroendocrine tumorigenesis, particularly in metastasis formation. In addition, the expression of RWDD3 was demonstrated to be increased in pituitary tumors, glioma and von Hippel-Lindau tumors, suggesting that it may contribute to the development and progression of these tumors (5,10-12). Recently, Fan et al (13) reported that inhibition of RWDD3 expression reduced glioblastoma U251 cells proliferation, migration and invasion, as well as induced cell apoptosis. However, the clinical significance of RWDD3 expression and the regulatory mechanism of RWDD3 in glioblastoma remain largely unknown.

Therefore, the present study investigated the expression of RWDD3 in glioblastoma tissues and cell lines, as well as 
its clinical significance. Furthermore, the study examined the exact roles of RWDD3 in regulating the proliferation, cell cycle progression, apoptosis, migration and invasion of glioblastoma cells in vitro, as well as the involved molecular mechanism.

\section{Materials and methods}

Clinical tissue samples. The present study was approved by the Ethics Committee of the Brain Hospital of Hunan Province (also known as the Second People's Hospital of Hunan Province, Changsha, China). A total of 63 glioblastoma tissues and 13 normal brain tissues were collected during surgical resection at the Second People's Hospital of Hunan Province between April 2011 and March 2014. The glioblastoma patients included 41 male and 32 female, with an age ranging between 32 and 62 years, and mean age of 43.2 years. These normal tissues were obtained from 13 patients ( 8 male and 5 female patients), with an age ranging between 38 and 57 years (mean age of 45.3 years). In addition, these glioblastoma patients were all at stage IV, and divided into a high RWDD3 expression group and a low expression group based on the calculated mean expression value (1.81 was the cut-off value). Written informed consents were obtained from all patients. The survival of these patients was recorded, and patients were followed up for 32 months. The tissue samples were frozen in liquid nitrogen immediately following surgery and stored at $-80^{\circ} \mathrm{C}$ until further use.

Cell culture and transfection. Human glioblastoma cell lines, including A172 (CRL-1620 $\left.{ }^{\mathrm{TM}}\right)$, U87 (HTB-14 ${ }^{\mathrm{TM}}$ ), U251 (accession number not found), M059J (CRL-2366 ${ }^{\mathrm{TM}}$ ) and T98G $\left(\mathrm{CRL}-1690^{\mathrm{TM}}\right)$, and normal human astrocytes were purchased from the American Type Culture Collection (Rockville, MD, USA). Cells were cultured in Dulbecco's modified Eagle's medium (DMEM; Thermo Fisher Scientific, Inc., Waltham, MA, USA) supplemented with $10 \%$ fetal bovine serum (FBS; Thermo Fisher Scientific, Inc.) at $37^{\circ} \mathrm{C}$ in a humidified incubator containing 5\% $\mathrm{CO}_{2}$. Cell transfection was conducted using Lipofectamine ${ }^{\circledR} 2000$ (Thermo Fisher Scientific, Inc.), according to the manufacturer's protocol. Briefly, RWDD3-specific small interfering RNA (RWDD3 siRNA, Santa Cruz Biotechnology, Inc., Dallas, TX, USA) was transfected into U87 and U251 cells to knockdown the RWDD3 expression. Besides, non-specific siRNA (NC siRNA, Santa Cruz Biotechnology, Inc.) was transfected into U87 and U251 cells as the control group. Following transfection for $48 \mathrm{~h}$, the expression levels of RWDD3 were examined. To clarify whether phosphoinositide 3-kinase/protein kinase B (PI3K/AKT) signaling was involved in RWDD3-mediated glioblastoma cells, 740Y-P (Fanbiotech, Beijing, China), an agonist of PI3K/AKT signaling, was used to treat RWDD3 siRNA-transfected U87 and U251 cells for $20 \mathrm{~min}$ at room temperature. Subsequent to the treatment, cell proliferation and invasion were evaluated.

Reverse transcription-quantitative polymerase chain reaction $(R T-q P C R)$. Total RNA was extracted from the glioblastoma tissues and cells using TRIzol ${ }^{\circledR}$ reagent (Thermo Fisher Scientific, Inc.) The RNA concentration was measured by using NanoDrop2000 (Thermo Fisher Scientific, Inc.), and then converted into cDNA using a Reverse Transcription kit (Thermo Fisher Scientific, Inc.), according to the manufacturer's protocol. For mRNA expression detection, qPCR was conducted using a One-Step qPCR kit (Toyobo Co., Ltd., Tokyo, Japan) on a 7500 thermocycler system (Thermo Fisher Scientific, Inc.), according to the manufacturer's instructions. The primer sequences used were as follows: RWDD3, 5'-AGA CAGATGGGACCGTGTTCA-3' (forward) and 5'-CTGCTT GCTCAAGTA ACTTCTCT-3' (reverse); GAPDH, 5'-GGA GCGAGATCCCTCCAAAAT-3' (forward) and 5'-GGC TGTTGTCATACTTCTCATGG-3' (reverse). The PCR conditions involved initial denaturation at $95^{\circ} \mathrm{C}$ for $5 \mathrm{~min}$, followed by 35 cycles of denaturation at $95^{\circ} \mathrm{C}$ for $15 \mathrm{sec}$, and annealing/elongation at $60^{\circ} \mathrm{C}$ for $30 \mathrm{sec}$. The experiment was repeated three times. The relative mRNA expression was analyzed by the $2^{-\Delta \Delta \mathrm{Cq}}$ method (14).

Western blot analysis. Cells were solubilized in cold radioimmunoprecipitation lysis buffer (Thermo Fisher Scientific, Inc.), and the concentration of protein was examined using a BCA Protein Assay kit (Pierce; Thermo Fisher Scientific, Inc.), according to the manufacturer's protocol. Next, $60 \mu \mathrm{g}$ protein per lane was separated by $12 \%$ SDS-PAGE and then transferred onto a polyvinylidene difluoride membrane (Thermo Fisher Scientific, Inc.). The membrane was subsequently incubated with phosphate-buffered saline (PBS) containing 5\% milk at room temperature for $3 \mathrm{~h}$. Following three washes with PBS, the membrane was incubated at room temperature for $3 \mathrm{~h}$ with the following rabbit primary antibodies: Polyclonal anti-RWDD3 (1:50; ab91555), polyclonal anti-matrix metalloproteinase 2 (MMP2; 1:100; ab37150), polyclonal anti-MMP9 (1:100; ab38898), polyclonal anti-B-cell lymphoma 2 (Bcl-2; 1:100; ab59348), polyclonal anti-Bcl-2-associated $\mathrm{X}$ protein (Bax; 1:100; ab53154), monoclonal anti-total PI3K (1:50; ab40755), polyclonal anti-phosphorylated PI3K (1:50; ab182651), polyclonal anti-total AKT (1:50; ab8805), polyclonal anti-phosphorylated AKT (1:50; ab38449) and polyclonal anti-GAPDH (1:50; ab9485; all purchased from Abcam, Cambridge, MA, USA). Subsequent to three washes with PBS, the membrane was incubated with horseradish peroxidase-conjugated goat anti-rabbit secondary antibody (1:5,000; ab6721; Abcam) at room temperature for $40 \mathrm{~min}$. After a further three washes with PBS, chemiluminescent detection was conducted using an Enhanced Chemiluminescence kit (Pierce; Thermo Fisher Scientific, Inc.), according to the manufacturer's protocol. The relative protein expression was analyzed using Image-Pro plus software 5.0 (Media Cybernetics, Inc., Rockville, MD, USA).

Cell proliferationassay. U87 and U251 cells ( 20,000 cells/well) were seeded in a 96-well plate, and $100 \mu 1$ DMEM containing $0.5 \mathrm{~g} / 1$ MTT (Thermo Fisher Scientific, Inc.) was added. Subsequent to culture at $37^{\circ} \mathrm{C}$ for $12,24,48$ or $72 \mathrm{~h}$, the medium was removed, and $50 \mu 1$ dimethyl sulfoxide (Beyotime Institute of Biotechnology, Shanghai, China) was added. Following incubation at $37^{\circ} \mathrm{C}$ for $10 \mathrm{~min}$, the absorbance was measured at a wavelength of $570 \mathrm{~nm}$ using the Varioskan LUX multimode microplate reader (Thermo Fisher Scientific, Inc.).

Cell apoptosis assay. Cell apoptosis was examined using the Alexa Fluor ${ }^{\circledR} 488$ Annexin V/Dead Cell Apoptosis 

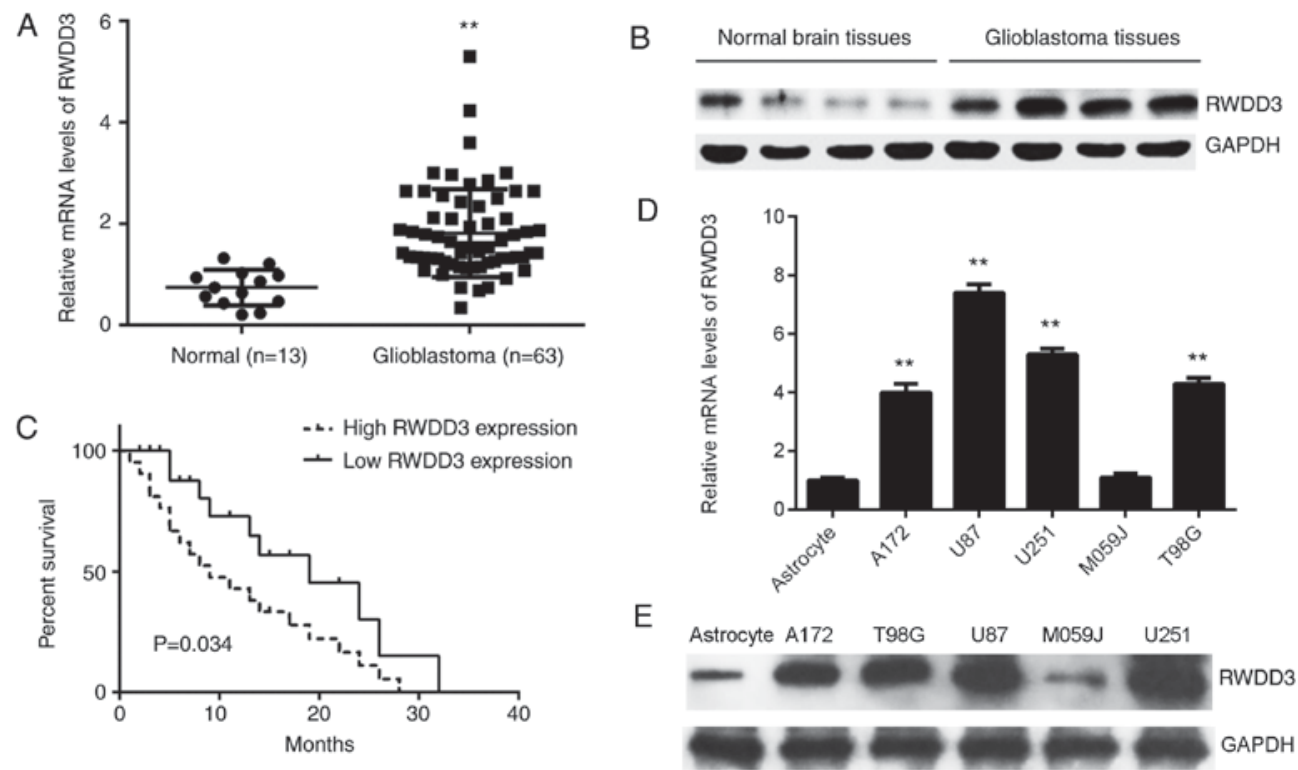

Figure 1. (A) mRNA and (B) protein expression levels of RWDD3 were significantly higher in glioblastoma tissues when compared with that in normal brain tissues. ${ }^{* *} \mathrm{P}<0.01$ vs. normal group. (C) Glioblastoma patients with high RWDD3 levels presented shorter overall survival time when compared with those with low RWDD3 levels. (D) mRNA and (E) protein expression levels of RWDD3 were significantly higher in glioblastoma cell lines when compared with that in normal human astrocytes. ${ }^{* *} \mathrm{P}<0.01$ vs. astrocyte. RWDD3, RWD domain containing 3.

kit (Thermo Fisher Scientific, Inc.), according to the manufacturer's protocol. Briefly, U87 and U251 cells were harvested and re-suspended in $200 \mu 1$ binding buffer. Next, cells $\left(1 \times 10^{6}\right.$ cells $\left./ \mathrm{ml}\right)$ were incubated with $10 \mu \mathrm{l}$ Annexin V and $5 \mu 1$ propidium iodide (PI) in the dark for $15 \mathrm{~min}$. Subsequently, $300 \mu 1$ binding buffer was added, and the cells were analyzed using BD Accuri C6 flow cytometer (BD Biosciences, Franklin Lakes, NJ, USA).

Cell cycle progression analysis. U87 and U251 cells (1x106) were washed twice with PBS, resuspended in $70 \%$ ethanol and fixed overnight at $-20^{\circ} \mathrm{C}$. The cells were washed twice in PBS with $3 \%$ bovine serum albumin (BSA) and then incubated at room temperature for $30 \mathrm{~min}$ in PBS containing $40 \mu \mathrm{g} / \mathrm{ml}$ PI, $3 \% \mathrm{BSA}$ and $0.2 \mathrm{mg} / \mathrm{ml}$ RNase. The DNA content was analyzed using the BD Accuri C6 flow cytometer.

Cell invasion assay. The cell invasion ability was evaluated using BD Falcon Cell Culture Inserts (BD Biosciences) pre-coated with Matrigel (BD Biosciences), according to the manufacturer's protocol. Briefly, the U87 and U251 cell suspension $\left(2 \times 10^{5}\right.$ cells $\left./ \mathrm{ml}\right)$ was prepared in DMEM, and $300 \mu \mathrm{l}$ of the suspension was added into the upper chamber of the 24-well plates, while $300 \mu 1$ DMEM supplemented with $10 \%$ FBS was added into the lower chamber. Following incubation at $37^{\circ} \mathrm{C}$ for $24 \mathrm{~h}$, cells that did not invade through the membrane in the filter were carefully wiped out using a cotton-tipped swab. Next, the filter was fixed in $90 \%$ alcohol at room temperature for $10 \mathrm{~min}$ and then stained with $0.1 \%$ crystal violet (Thermo Fisher Scientific, Inc.) at room temperature for $10 \mathrm{~min}$. The invading cells were counted under a BX53 microscope (Olympus Corp., Tokyo, Japan).

Cell migration assay. U87 and U251 cells were cultured to $100 \%$ confluence. In order to terminate cell proliferation, mitomycin C (10 $\mu \mathrm{g} / \mathrm{ml}$; Sigma-Aldrich; Merck KGaA, Darmstadt, Germany) was used to treat the cells at $37^{\circ} \mathrm{C}$ for $2 \mathrm{~h}$. The cells were then washed with DMEM three times, and wounds with a width of $\sim 1 \mathrm{~mm}$ were created with a plastic scriber. Subsequent to washing with PBS, the cells were incubated in DMEM supplemented with $10 \%$ FBS at $37^{\circ} \mathrm{C}$ for $48 \mathrm{~h}$. Finally, the cells were observed under the BX53 microscope.

Statistical analysis. Data are presented as the mean \pm standard deviation of at least three samples. SPSS version 17.0 software (SPSS, Inc., Chicago, IL, USA) was used to conduct statistical analyses. Differences were examined using Student's t-test for two-group comparison or analysis of variance for comparison of more than two groups. The Kaplan-Meier method was used for survival analysis. $\mathrm{P}<0.05$ was considered to indicate a statistically significant difference.

\section{Results}

Upregulation of RWDD3 in human glioblastoma tissues. To preliminarily investigate the role of RWDD3 in glioblastoma, the current study first examined the mRNA and protein expression levels of RWDD3 in glioblastoma and normal brain tissues. As shown in Fig. 1A and B, the mRNA and protein expression levels of RWDD3 were significantly higher when compared with those in normal brain tissues. These patients were all at stage IV, and divided into a high RWDD3 expression group and a low expression group based on the mean expression value (1.81 was the cut-off value). In addition, the patients with high RWDD3 levels presented a shorter overall survival time when compared with those with low RWDD3 levels (Fig. 1C). Subsequently, the expression of RWDD3 in certain common glioblastoma cell lines was examined, including in A172, U87, U251, M059J and T98G cells. As shown in Fig. 1D and E, the mRNA and protein levels of RWDD3 were also increased in 
A

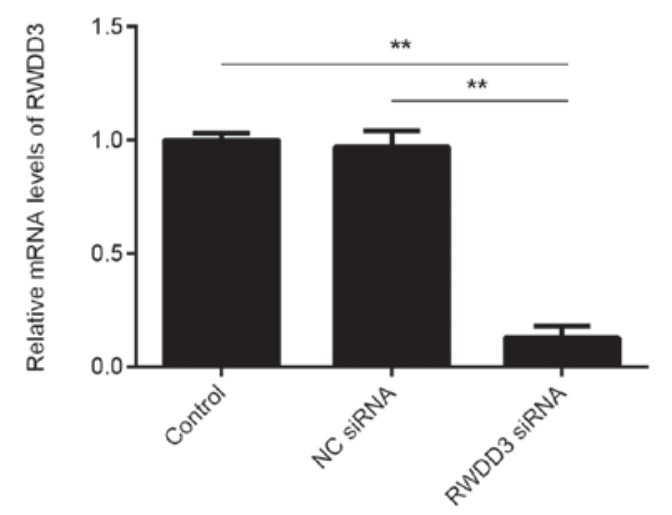

B

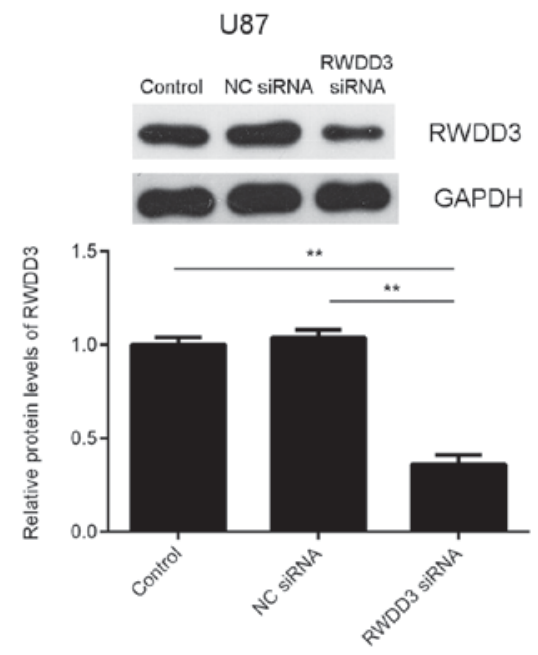

C

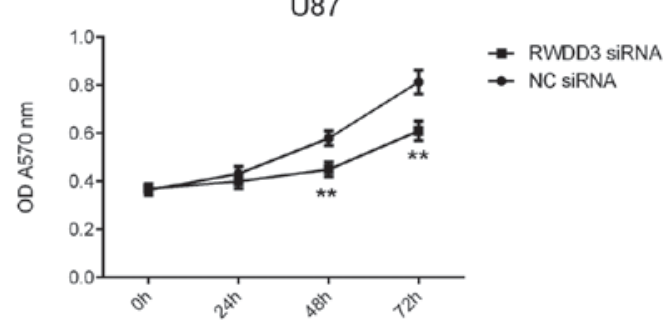

D
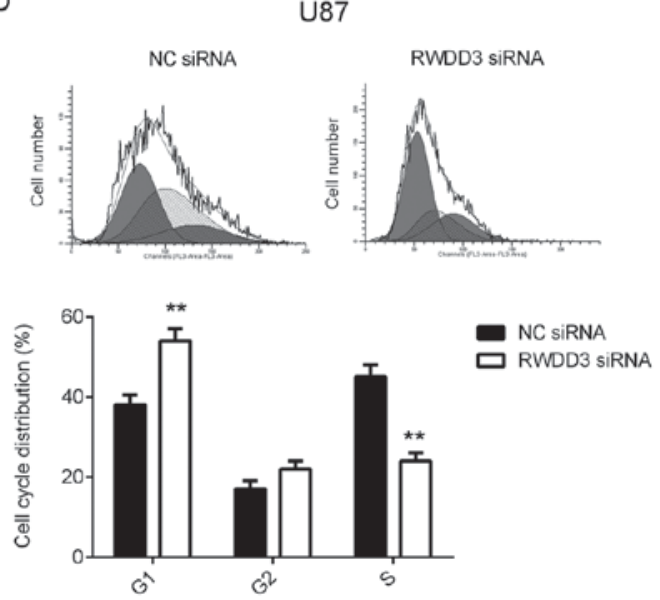

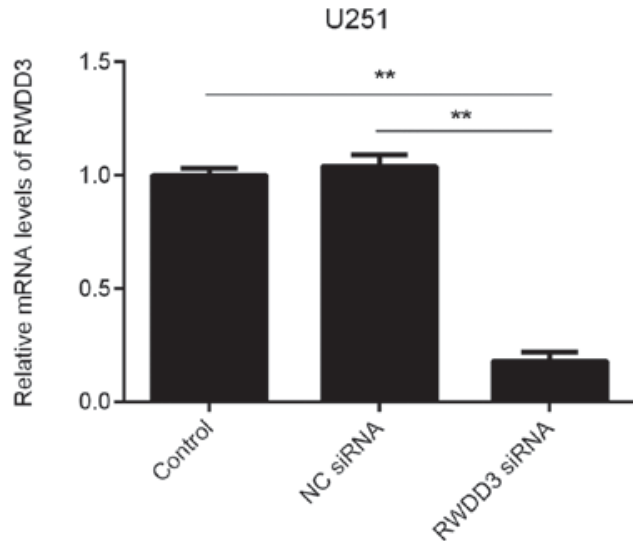

U251

Control NC SiRNA RWDDA

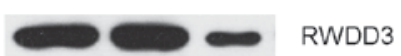

$\longrightarrow$ GAPDH

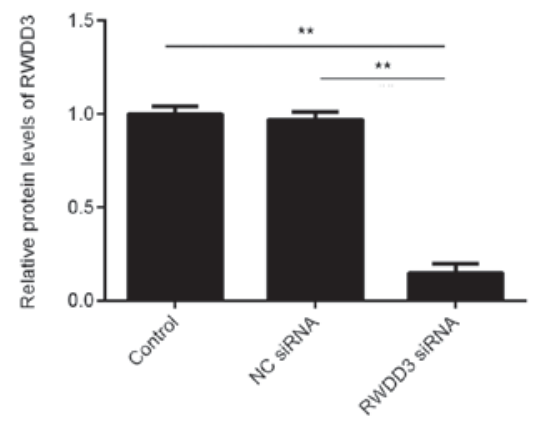

U251

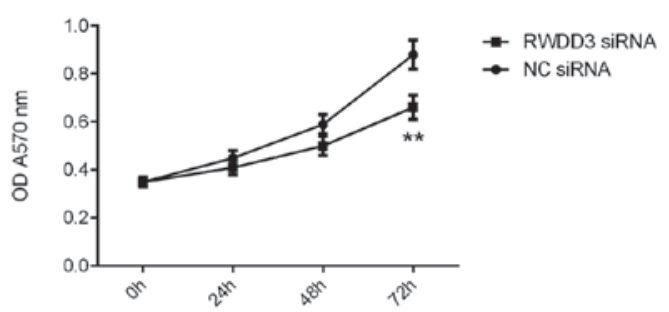

U251

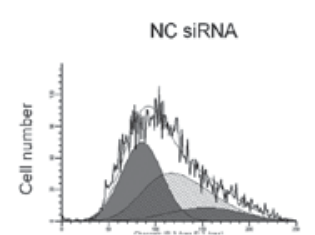

RWDD3 siRNA
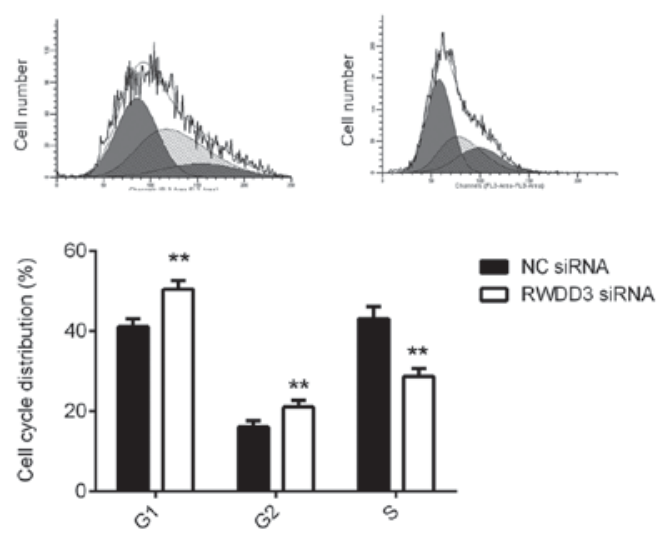

Figure 2. U87 and U251 cells were transfected with RWDD3 siRNA or NC siRNA. (A) Reverse transcription-quantitative polymerase chain reaction and (B) western blot analysis were used to examine the mRNA and protein levels of RWDD3, respectively. ${ }^{* *} \mathrm{P}<0.01$ as indicated. (C) MTT assay and (D) flow cytometry were used to examine cell proliferation and cell cycle distribution, respectively. ${ }^{*} \mathrm{P}<0.01$ vs. NC siRNA group. RWDD3, RWD domain containing 3; siRNA, small interfering RNA; NC, negative control; OD, optical density. 
A

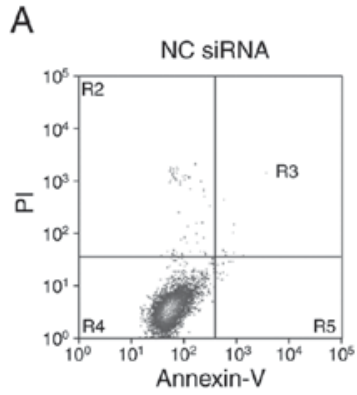

U87
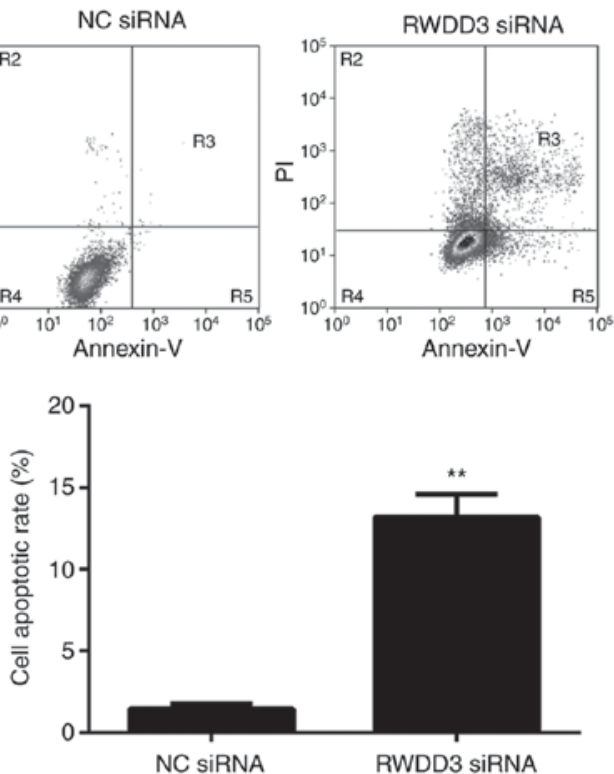

B
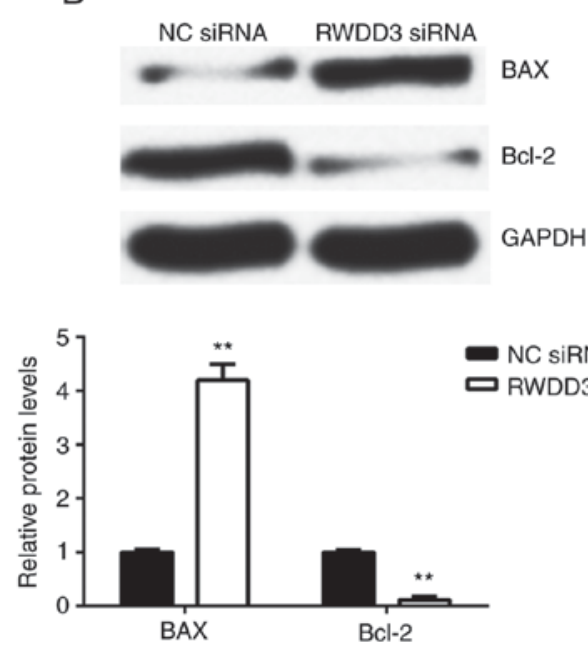

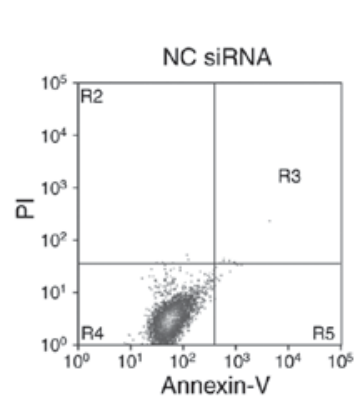

U251
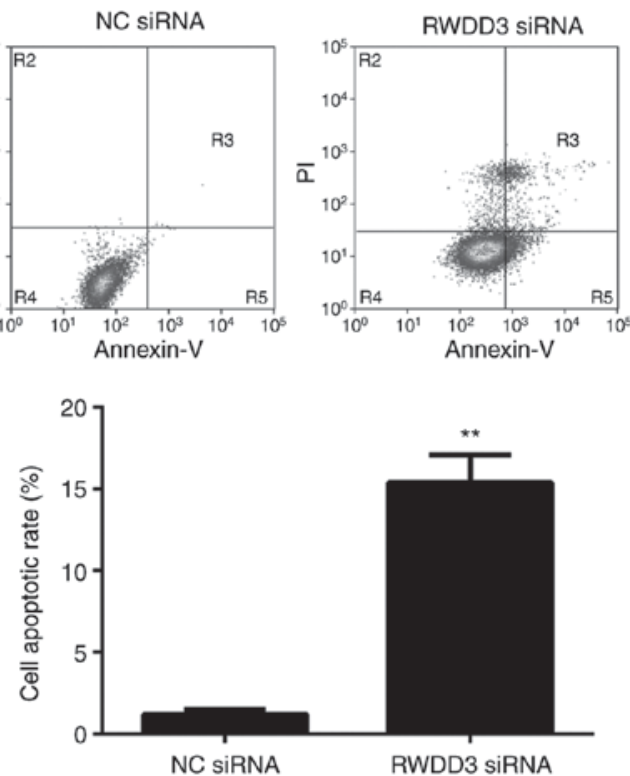

U251
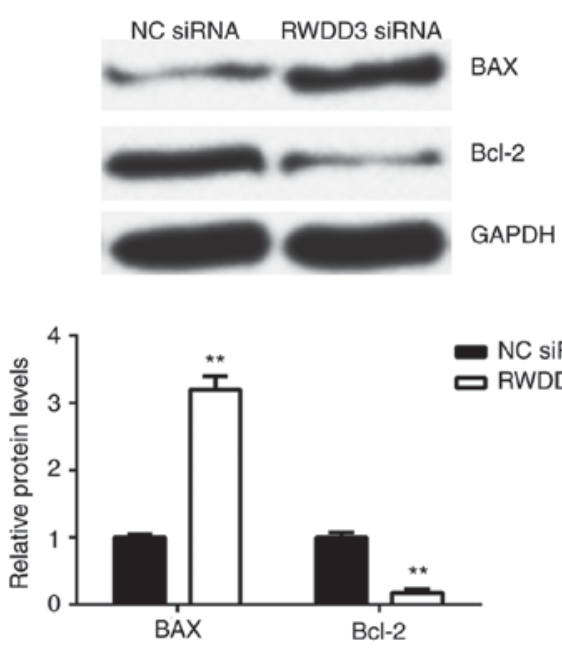

Figure 3. U87 and U251 cells were transfected with RWDD3 siRNA or NC siRNA. (A) Flow cytometry were used to examine cell apoptosis. (B) Western blot analysis was used to examine the levels of apoptosis-associated proteins, Bax and Bcl-2. ${ }^{* *} \mathrm{P}<0.01$ vs. NC siRNA group. RWDD3, RWD domain containing 3; siRNA, small interfering RNA; NC, negative control; Bcl-2, B-cell lymphoma 2; Bax, Bcl-2-associated X protein.

glioblastoma cells compared with the normal human astrocyte cells. Therefore, it is suggested that the increased expression of RWDD3 may be associated with the malignancy of glioblastoma.

Knockdown of RWDD3 suppresses glioblastoma cell proliferation and induces cell cycle arrest at G1 phase. As U87 and U251 cells exhibited the highest expression of RWDD3, these two cell lines were used in the subsequent experiments in vitro. In order to downregulate the RWDD3 levels, U87 and U251 cells were transfected with RWDD3 siRNA. Following transfection, the mRNA and protein levels of RWDD3 were significantly reduced in RWDD3 siRNA group compared with control group (Fig. 2A and B). However, transfection with negative control (NC) siRNA exerted no evident effect on the expression of RWDD3 (Fig. 2A and B).

Next, an MTT assay was conducted to determine the effects of RWDD3 downregulation on the proliferation of U87 and U251 cells. As shown in Fig. 2C, knockdown of RWDD3 significantly inhibited $\mathrm{U} 87$ at 48 and $72 \mathrm{~h}$ and $\mathrm{U} 251$ cell proliferation at $72 \mathrm{~h}$ when compared with the NC siRNA group. The cell cycle distribution was also further examined, and it was observed that inhibition of RWDD3 expression led to a cell cycle arrest at $G_{1}$ and $G_{2}$ phase (Fig. 2D). Therefore, these findings suggested that knockdown of RWDD3 suppressed glioblastoma cell proliferation, at least partly, via inducing a cell cycle arrest at $G_{1}$ phase.

RWDD3 downregulation promotes glioblastoma cell apoptosis. The effect of RWDD3 knockdown on glioblastoma cell apoptosis was further analyzed. As shown in Fig. 3A, the cell apoptosis was significantly increased in the RWDD3-downregulated U87 and U251 cells, when compared with the NC siRNA group. The present study then examined the expression of apoptotic-associated genes, including $\mathrm{Bcl}-2$ and Bax. The data indicated that the 
A

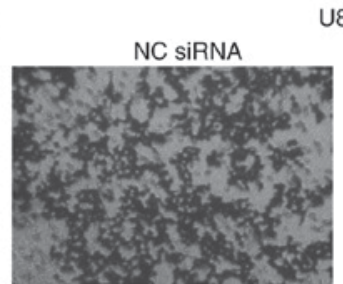

U87
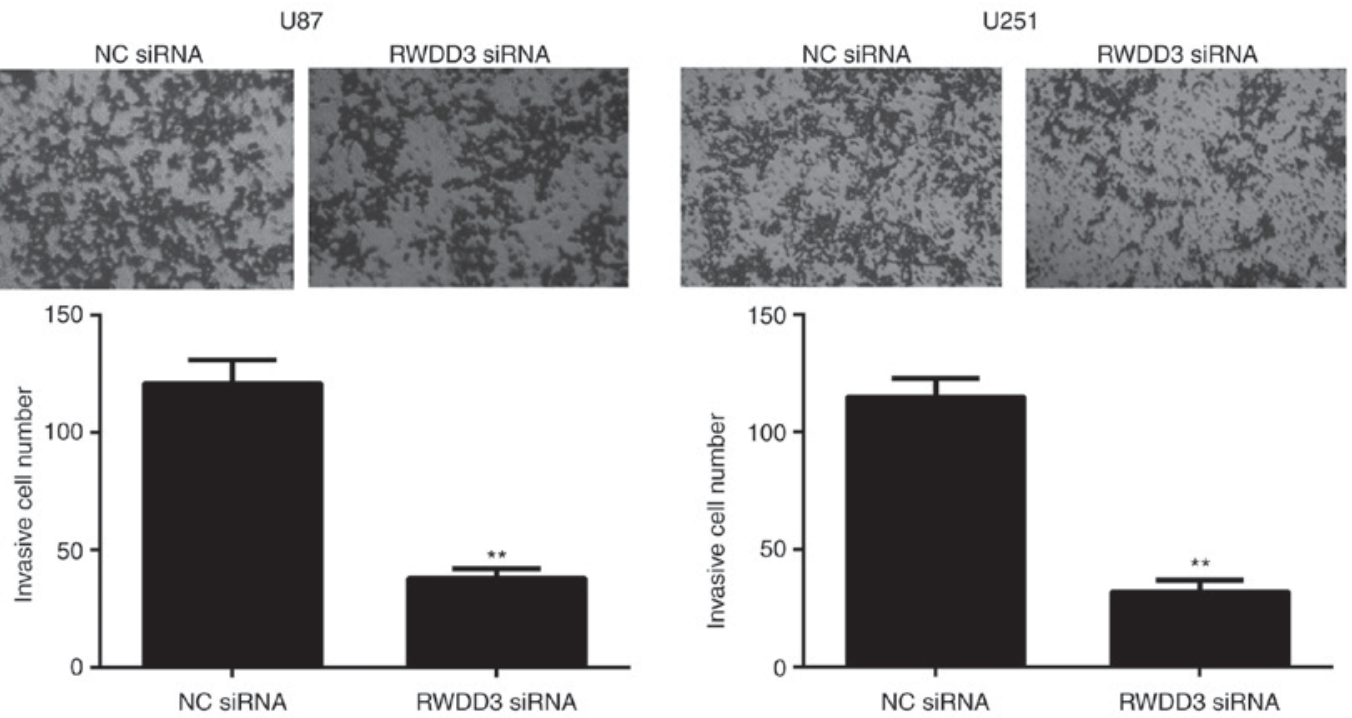

B
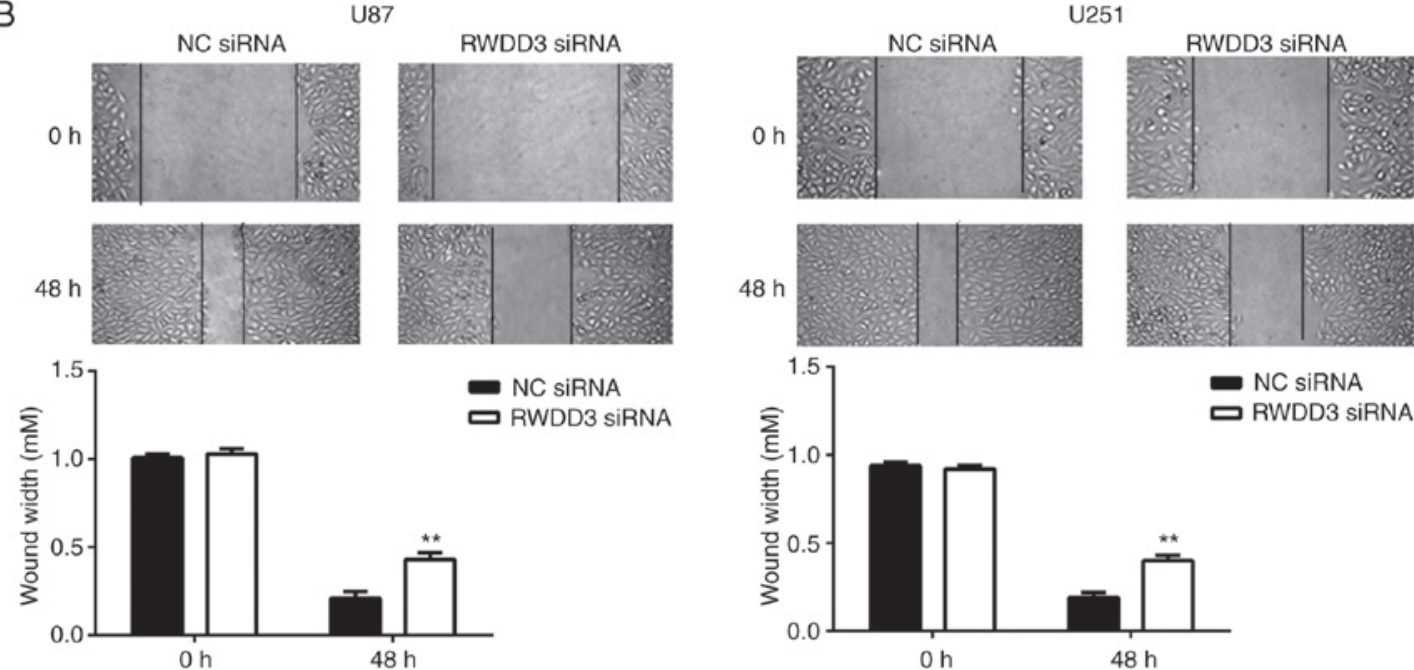

- NC SiRNA

RWDD3 siRNA
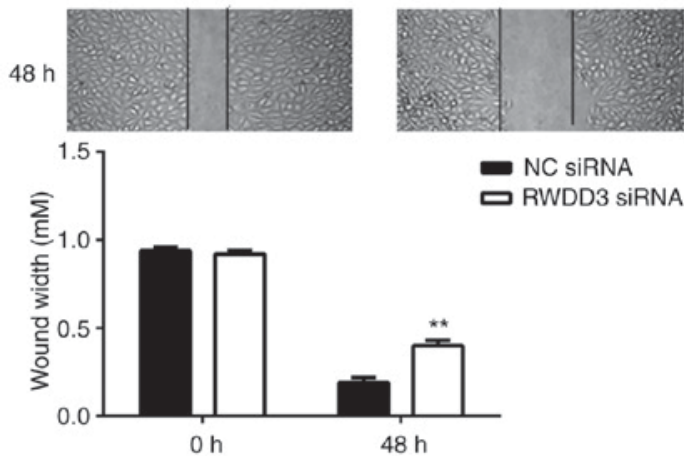

nC SiRNA

RWDD3 SiRNA

C

U87

U251

NC SIRNA RWDD3 SiRNA

NC siRNA RWDD3 SiRNA
$-\mathrm{MMP2}$
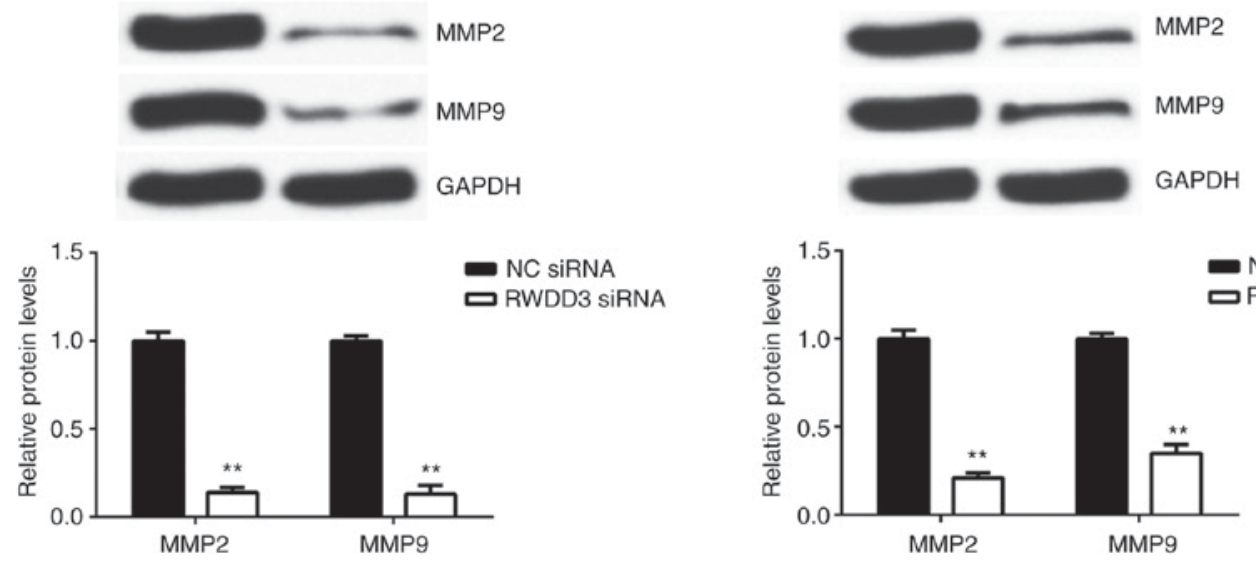

Figure 4. U87 and U251 cells were transfected with RWDD3 siRNA or NC siRNA. (A) Transwell assay and (B) wound healing assay were used to examine the cell invasion and migration, respectively. (C) Western blot analysis was used to examine the protein levels of MMP2 and MMP9. ${ }^{* * *} \mathrm{P}<0.01$ vs. NC siRNA group. RWDD3, RWD domain containing 3; siRNA, small interfering RNA; NC, negative control; MMP, matrix metalloproteinase.

protein expression of the pro-apoptotic Bax was significantly upregulated, while the protein expression of anti-apoptotic Bcl-2 was markedly downregulated, in U87 and U251 cells following the knockdown of RWDD3 expression by siRNA transfection (Fig. 3B). These data suggest that RWDD3 may inhibit glioblastoma cell apoptosis via affecting the expression levels of Bax and Bcl-2.

Downregulation of RWDD3 reduces U87 and U251 cell invasion and migration. The invasion and migration capacities 

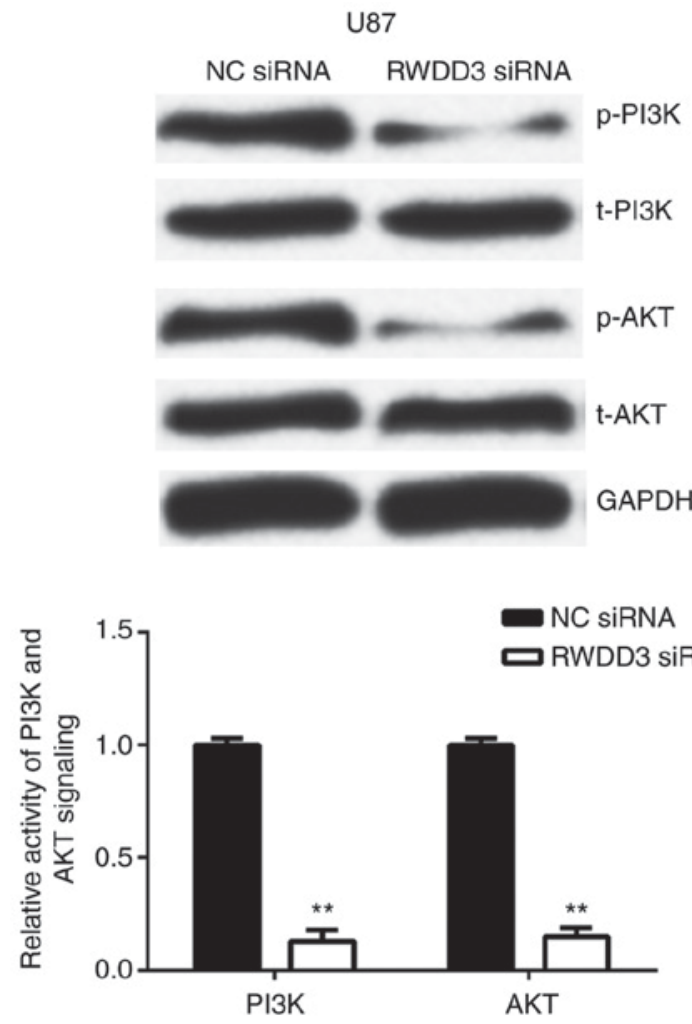

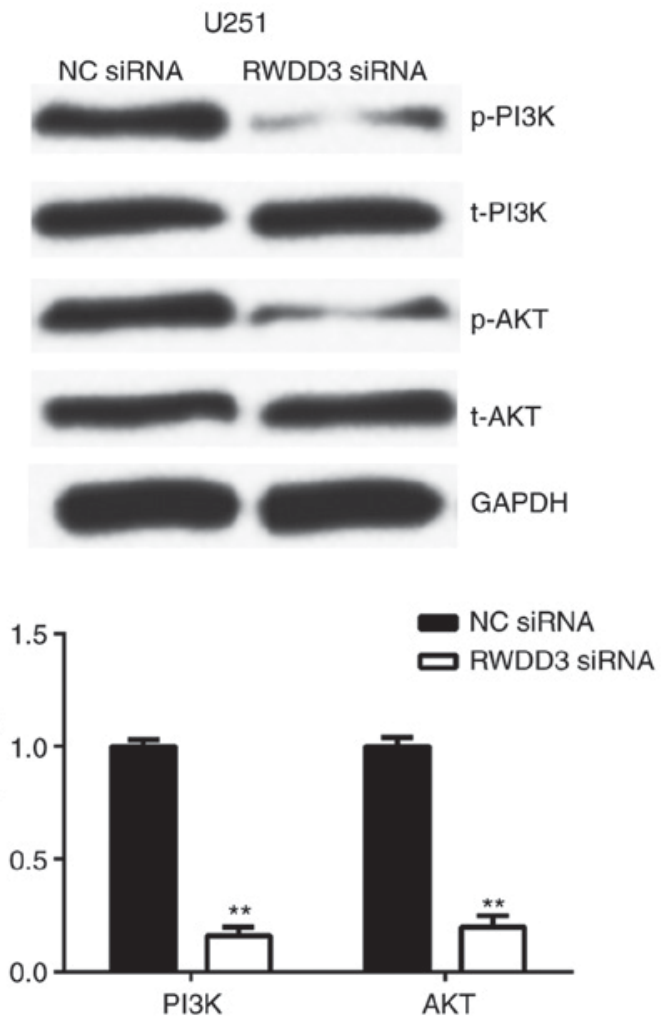

Figure 5. U87 and U251 cells were transfected with RWDD3 siRNA or NC siRNA. Western blot analysis was conducted to examine the phosphorylation levels of PI3K and AKT. ${ }^{* *} \mathrm{P}<0.01$ vs. NC siRNA group. RWDD3, RWD domain containing 3; siRNA, small interfering RNA; NC, negative control; PI3K, phosphoinositide 3-kinase; AKT, protein kinase B; t-, total; p-, phosphorylated.

of U87 and U251 cells following the knockdown of RWDD3 were also examined. As shown in Fig. 4A and B, transfection with RWDD3 siRNA caused a significant reduction in the invasion and migration of U87 and U251 cells, when compared with the NC siRNA group. Further investigation revealed that the protein levels of MMP2 and MMP9 were also markedly decreased following the inhibition of RWDD3 in U87 and U251 cells (Fig. 4C). These findings suggest that RWDD3 serves a promoting role in glioblastoma cell invasion and migration via mediating the MMP2 and MMP9 expression levels.

Inhibition of RWDD3 expression downregulates PI3K/AKT signaling in U87 and U251 cells. The PI3K/AKT signaling pathway has been demonstrated to be crucial for the survival, proliferation and motility of multiple types of cells $(15,16)$. Thus, the present study subsequently examined the activity of the PI3K/AKT signaling pathway in U87 and U251 cells following inhibition of RWDD3 expression. As shown in Fig. 5, the phosphorylation levels of PI3K and AKT were significantly downregulated in U87 and U251 cells in the RWDD3 siRNA group, when compared with those in the NC siRNA group. These finding suggest that the promoting effects of RWDD3 on the malignant phenotypes of glioblastoma cells were exerted, partly at least, via affecting the activity of the PI3K/AKT signals.

To further confirm these findings, 740Y-P was used, which is an agonist of PI3K/AKT signaling, to further investigate whether the cell proliferation or migration could be restored. The findings demonstrated that the proliferation and migration were significantly upregulated in the RWDD3
siRNA + 740Y-P group, when compared with the RWDD3 siRNA group (Fig. 6A and B), suggesting that the activation of PI3K/AKT signaling rescued the suppressive effects of RWDD3 downregulation on glioblastoma cell proliferation and migration. Consistently, the MMP2 and MMP9 protein levels were also increased in the RWDD3 siRNA + 740Y-P group, when compared with the RWDD3 siRNA group (Fig. 6C).

\section{Discussion}

Limited evidence exists regarding the clinical significance of RWDD3 expression and the regulatory mechanism of RWDD3 in glioblastoma. Thus, this was examined in the present study. It was initially observed that RWDD3 was significantly upregulated in glioblastoma tissues, and a high expression of RWDD3 was associated with poor prognosis of patients. Subsequently, knockdown of RWDD3 in glioblastoma cell lines significantly reduced the cell proliferation, cell cycle progression, migration and invasion, whereas it promoted cell apoptosis, accompanied with reduced activity of PI3K/AKT signaling. Furthermore, activation of PI3K/AKT signaling rescued the suppressive effects of RWDD3 downregulation on glioblastoma cell proliferation and migration, concurrent with increased protein levels of MMP2 and MMP9.

During the development and malignant progression of glioblastoma, metabolic reorganization is driven by HIF-1 $\alpha$, which makes use of aerobic glycolysis as the main source of energy and biosynthetic molecules $(17,18)$. It has been demonstrated that HIF-1 $\alpha$ promoted the expression of numerous 
A

U87

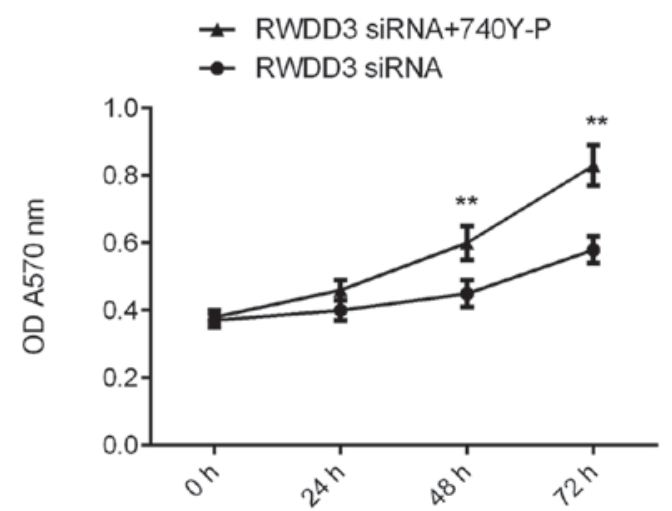

B

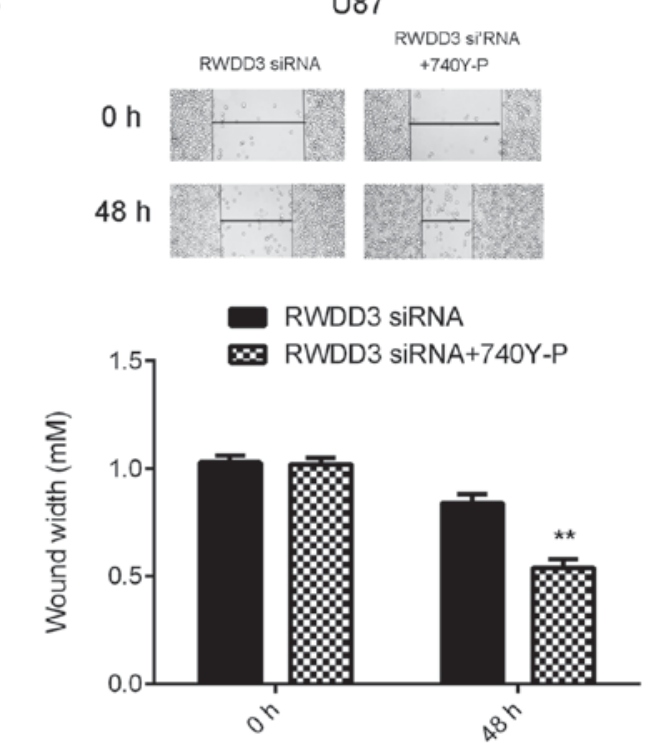

C

U87
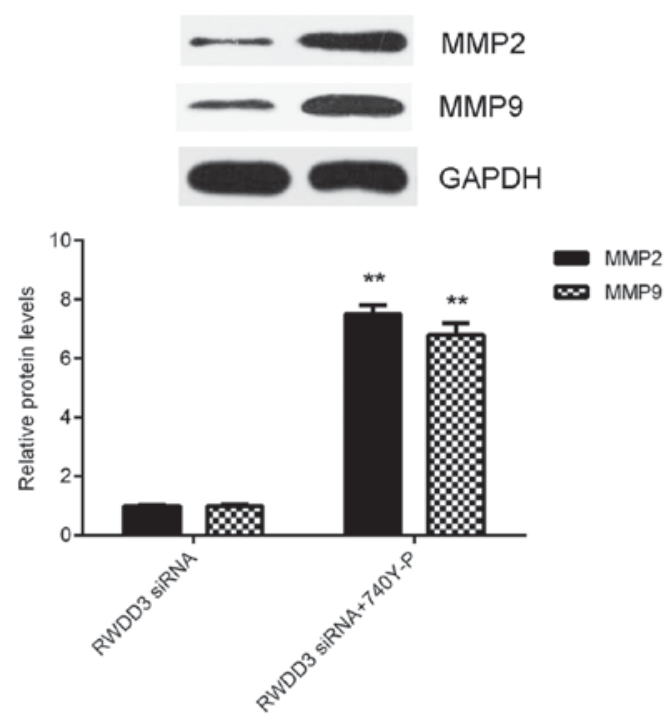

U251

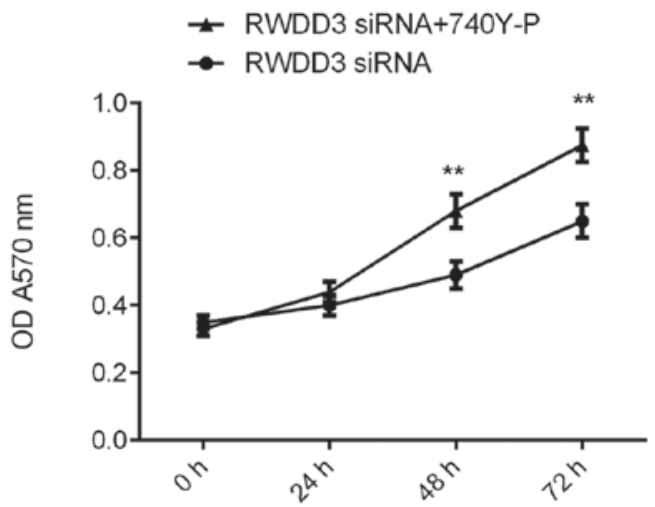

U251

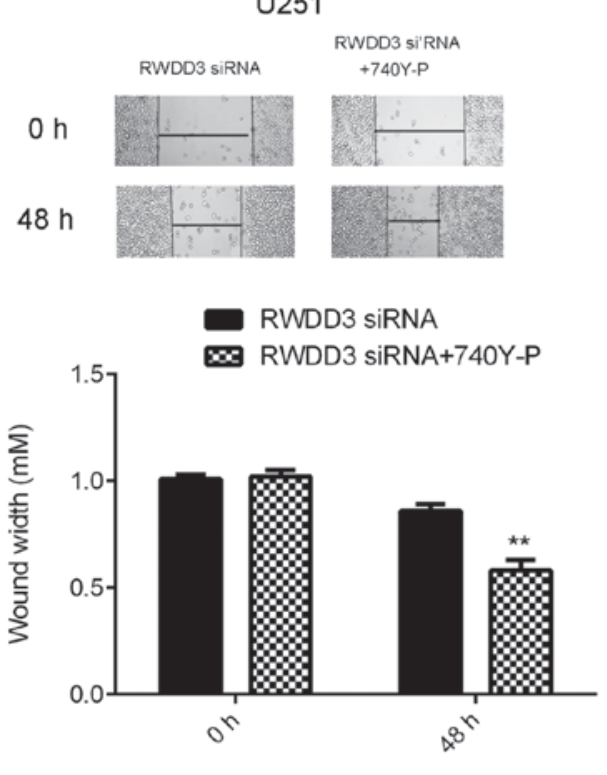

U251
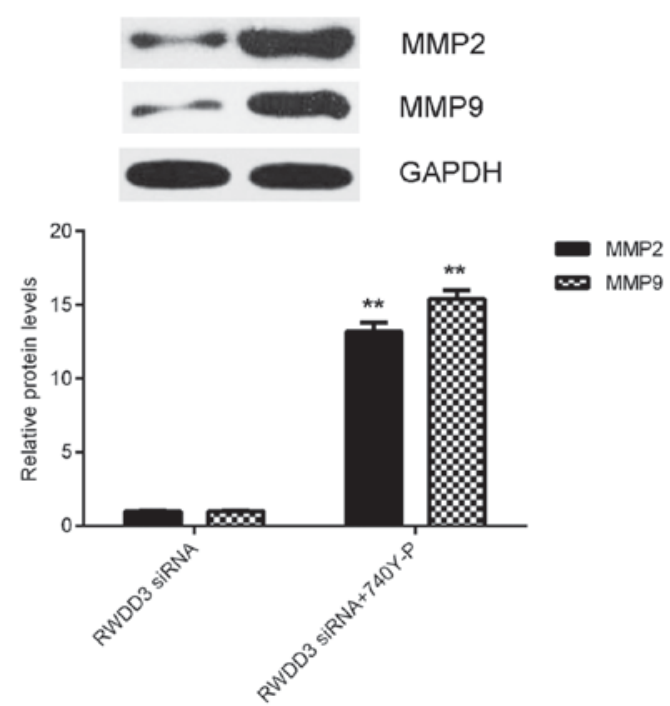

Figure 6. RWDD3 siRNA-transfected U87 and U251 cells were treated with or without 740Y-P. (A) MTT assay and (B) wound healing assay were conducted to examine the cell proliferation and migration, respectively, following treatment for $2 \mathrm{~h}$. (C) Western blot analysis was used to examine the protein levels of MMP2 and MMP9. * $\mathrm{P}<0.01$ vs. RWDD3 siRNA group. RWDD3, RWD domain containing 3; siRNA, small interfering RNA; MMP, matrix metalloproteinase.

genes associated with tumor growth and metastasis (19-21). The expression and activity of HIF-1 $\alpha$ is controlled mainly by oxygen levels (8). Under normoxia, HIF-1 $\alpha$ rapidly binds oxygen-dependent ubiquitin, which leads to subsequent 
degradation of HIF-1 $\alpha$ (8). However, SUMO-1, which has a similar structure to that of ubiquitin, competes with ubiquitin for HIF-1 $\alpha$ and thus protects HIF-1 $\alpha$ from degradation (22). Previous studies have demonstrated that RWDD3 is able to enhance the stabilization and transcriptional activity of HIF-1 $\alpha$ during hypoxia via promoting the sumoylation of HIF-1 $\alpha$, through which RWDD3 may serve a promoting role in tumor growth and metastasis $(8,10)$. In addition, it has been reported that RWDD3 was upregulated in glioma, as well as in pituitary tumors $(10,23)$; however, the clinical significance of RWDD3 expression and the regulatory mechanism of RWDD3 in glioblastoma requires further elucidation. In the present study, it was initially demonstrated that RWDD3 was upregulated in glioblastoma tissues, and this upregulation may predicate poor prognosis in glioblastoma patients. Consistent with the clinical data, the expression levels of RWDD3 were also significantly increased in several common glioblastoma cell lines, when compared with normal human astrocyte cells. Therefore, the current study reported for the first time an upregulation of RWDD3 in glioblastoma.

The present study further examined the function of RWDD3 in the regulation of the malignant phenotypes of glioblastoma cells in vitro using U87 and U251 cells, as they exhibited the highest expression of RWDD3. It was observed that knockdown of RWDD3 caused a significant reduction in U87 and U251 cell proliferation. This reduced cell proliferation caused by RWDD3 downregulation may be attributed to the cell cycle arrest at G1 phase and cell apoptosis. To further confirm these findings, the study examined the expression levels of apoptosis-associated proteins, Bax and Bcl-2. It was observed that the pro-apoptotic Bax was significantly upregulated following RWDD3 knockdown, while the anti-apoptotic $\mathrm{Bcl}-2$ was markedly downregulated following inhibition of RWDD3 expression in glioblastoma cells.

MMP2 and MMP9 are zinc-dependent enzymes capable of cleaving type IV and V collagens $(24,25)$. Through this function, they participate in the breakdown of the extracellular matrix in physiological processes, including embryonic development, reproduction and tissue remodeling, as well as in pathological processes, such as tumor cell invasion and migration $(26,27)$. In the present study, it was identified that inhibition of RWDD3 expression led to reduced glioblastoma cell migration and invasion, accompanied with decreased protein levels of MMP2 and MMP9. These findings suggest that RWDD3 was able to mediate the protein expression of MMP2 and MMP9, and thus served a promoting role in glioblastoma cell migration and invasion.

PI3K/AKT signaling has a central role in the regulation of tumor cell survival, growth, motility, angiogenesis and metabolism (28). Previous studies have demonstrated that the $\mathrm{PI} 3 \mathrm{~K} / \mathrm{AKT}$ signaling is frequently hyperactivated in various types of human cancer, including glioblastoma, and is thus suggested to be a potential therapeutic target for cancer treatment $(29,30)$. Indeed, several PI3K inhibitors have been used in phase I/II clinical trials for glioblastoma treatment (30). In the current study, it was observed that knockdown of RWDD3 caused a decreased activity of PI3K/AKT signaling in U87 and U251 cells, while activation of PI3K/AKT signaling rescued the suppressive effects of RWDD3 downregulation on glioblastoma cell proliferation and migration, suggesting that the PI3K/AKT signaling is involved in the RWDD3-mediated malignant phenotypes of glioblastoma cells.

In conclusion, to the best of our knowledge, the present study is the first to demonstrate that RWDD3 is significantly upregulated in glioblastoma, and that it serves an oncogenic role in the regulation of the malignant phenotypes of glioblastoma cells, at least partly, via mediating the activity of PI3K/AKT signaling. Therefore, the current study suggests that RWDD3 may be a novel promising target for the treatment of glioblastoma. A limitation of the present study is the lack of in vivo experiments, and animal experiments should be conducted in future studies to examine the role of RWDD3 in glioblastoma.

\section{Acknowledgements}

Not applicable.

\section{Funding}

No funding was received.

\section{Availability of data and materials}

All data generated or analyzed during this study are included in this published article.

\section{Authors' contributions}

$\mathrm{XC}$ collected clinical tissues and wrote the manuscript. WK designed the study and revised the manuscript. HH, BL, YZ, $\mathrm{BZ}$ and LY performed the in vitro experiments.

\section{Ethics approval and consent to participate}

The present study was approved by the Ethics Committee of the Brain Hospital of Hunan Province (also known as the Second People's Hospital of Hunan Province, Changsha, China). Written informed consent was obtained from all patients.

\section{Consent for publication}

Written informed consents were obtained from all patients involved in the present study.

\section{Competing interests}

The authors declare that they have no competing interests.

\section{References}

1. Siegel RL, Miller KD and Jemal A: Cancer statistics, 2015. CA Cancer J Clin 65: 5-29, 2015.

2. Li Z, Guo J, Ma Y, Lin Z and Zhang L: Oncogenic role of MicroRNA-30b-5p in glioblastoma through targeting proline-rich transmembrane protein 2. Oncol Res: May 17, 2017 (Epub ahead of print).

3. Ding B, Cui B, Gao M, Li Z, Xu C, Fan S and He W: Knockdown of ras-related protein 25 ( $R$ ab25) inhibits the in vitro cytotoxicity and in vivo antitumor activity of human glioblastoma multiforme cells. Oncol Res 25: 331-340, 2017. 
4. Liu J, Li W, Liu S, Zheng X, Shi L, Zhang W and Yang H: Knockdown of collagen triple helix repeat containing 1 (CTHRC1) inhibits epithelial-mesenchymal transition and cellular migration in glioblastoma cells. Oncol Res 25: 225-232, 2017.

5. Antico Arciuch VG, Tedesco L, Fuertes M and Arzt E: Role of RSUME in inflammation and cancer. FEBS Lett 589: 3330-3335, 2015.

6. Liberman AC, Druker J, Garcia FA, Holsboer F and Arzt E: Intracellular molecular signaling. Basis for specificity to glucocorticoid anti-inflammatory actions. Ann N Y Acad Sci 1153: 6-13, 2009.

7. Druker J, Liberman AC, Antunica-Noguerol M, Gerez J, Paez-Pereda M, Rein T, Iñiguez-Lluhí JA, Holsboer F and Arzt E: RSUME enhances glucocorticoid receptor SUMOylation and transcriptional activity. Mol Cell Biol 33: 2116-2127, 2013.

8. Carbia-Nagashima A, Gerez J, Perez-Castro C, Paez-Pereda M, Silberstein S, Stalla GK, Holsboer F and Arzt E: RSUME, a small RWD-containing protein, enhances SUMO conjugation and stabilizes HIF-1alpha during hypoxia. Cell 131: 309-323, 2007.

9. Wu Y, Tedesco L, Lucia K, Schlitter AM, Garcia JM, Esposito I, Auernhammer CJ, Theodoropoulou M, Arzt E, Renner U and Stalla GK: RSUME is implicated in tumorigenesis and metastasis of pancreatic neuroendocrine tumors. Oncotarget 7: 57878-57893, 2016.

10. Fuertes M, Gerez J, Haedo M, Giacomini D, Páez-Pereda M, Labeur M, Stalla GK and Arzt E: Cytokines and genes in pituitary tumorigenesis: RSUME role in cell biology. Front Horm Res 38: 1-6, 2010.

11. Shan B, Gerez J, Haedo M, Fuertes M, Theodoropoulou M, Buchfelder M, Losa M, Stalla GK, Arzt E and Renner U: RSUME is implicated in HIF-1-induced VEGF-A production in pituitary tumour cells. Endocr Relat Cancer 19: 13-27, 2012.

12. Gerez J, Tedesco L, Bonfiglio JJ, Fuertes M, Barontini M, Silberstein S, Wu Y, Renner U, Páez-Pereda M, Holsboer F, et al: RSUME inhibits VHL and regulates its tumor suppressor function. Oncogene 34: 4855-4866, 2015.

13. Fan YH, Zhu XG, WU MJ, Chai Y, YE MH, Xi-Ao B and Wu L: Effects of lentivirus-mediated RWDD3 silencing on proliferation and invasion of human glioma U251 cells. Chin J Pathophysiol 31: $1550-1556,2015$.

14. Livak KJ and Schmittgen TD: Analysis of relative gene expression data using real-time quantitative PCR and the 2(-Delta Delta C(T)) method. Methods 25: 402-408, 2001

15. Katase N, Nishimatsu SI, Yamauchi A, Yamamura M, Terada K, Itadani M, Okada N, Hassan NMM, Nagatsuka H, Ikeda T, et al DKK3 overexpression increases malignant properties of head and neck squamous cell carcinoma cells. Oncol Res 26: 45-58, 2018.

16. Zhao P, Guan HT, Dai ZJ, Ma YG, Liu XX and Wang XJ: Knockdown of SPOCK1 inhibits the proliferation and invasion in colorectal cancer cells by suppressing the PI3K/Akt pathway. Oncol Res 24: 437-445, 2016.
17. Wu W, Hu Q, Nie E, Yu T, Wu Y,Zhi T, Jiang K, Shen F, Wang Y, Zhang J and You Y: Hypoxia induces H19 expression through direct and indirect Hif-1 $\alpha$ activity, promoting oncogenic effects in glioblastoma. Sci Rep 7: 45029, 2017.

18. Gabriely G, Wheeler MA, Takenaka MC and Quintana FJ: Role of AHR and HIF-1 $\alpha$ in glioblastoma metabolism. Trends Endocrinol Metab 28: 428-436, 2017.

19. Liu M, Wang D and Li N: MicroRNA-20b downregulates HIF-1a and Inhibits the proliferation and invasion of osteosarcoma cells. Oncol Res 23: 257-266, 2016.

20. Zhou Y, Yang C, Wang K, Liu X and Liu Q: MicroRNA-33b inhibits the proliferation and migration of osteosarcoma cells via targeting hypoxia-inducible factor-1 $\alpha$. Oncol Res 25: 397-405, 2017.

21. Liu F, Lin B, Liu X, Zhang W, Zhang E, Hu L, Ma Y, Li X and Tang X: ERK signaling pathway is involved in HPV-16 E6 but not E7 oncoprotein-induced HIF- $1 \alpha$ protein accumulation in NSCLC cells. Oncol Res 23: 109-118, 2016.

22. Wang F, Cai F, Shi R, Wei JN and Wu XT: Hypoxia regulates sumoylation pathways in intervertebral disc cells: Implications for hypoxic adaptations. Osteoarthritis Cartilage 24: 1113-1124, 2016.

23. Gerez J, Fuertes M, Tedesco L, Silberstein S, Sevlever G, Paez-Pereda M, Holsboer F, Turjanski AG and Arzt E: In silico structural and functional characterization of the RSUME splice variants. PLoS One 8: e57795, 2013.

24. Xue Q, Cao L, Chen XY, Zhao J, Gao L, Li SZ and Fei Z: High expression of MMP9 in glioma affects cell proliferation and is associated with patient survival rates. Oncol Lett 13: 1325-1330, 2017.

25. Zhu Y, Zhang X, Wang L, Ji Z, Xie M, Zhou X, Liu Z, Shi H and Yu R: Loss of SH3GL2 promotes the migration and invasion behaviours of glioblastoma cells through activating the STAT3/MMP2 signalling. J Cell Mol Med 21: 2685-2694, 2017.

26. Miao Y, Lu M, Yan Q, Li S and Feng Y: Inhibition of proliferation, migration, and invasion by knockdown of pyruvate kinase-M2 (PKM2) in ovarian cancer SKOV3 and OVCAR3 cells. Oncol Res 24: 463-475, 2016.

27. Amar S, Minond D and Fields GB: Clinical implications of compounds designed to inhibit ECM-modifying metalloproteinases. Proteomics 17, Issue 23-24, 2017.

28. Zhang J, Yu XH, Yan YG, Wang C and Wang WJ: PI3K/Akt signaling in osteosarcoma. Clin Chim Acta 444: 182-192, 2015.

29. Ramezani S, Vousooghi N, Ramezani Kapourchali F and Joghataei MT: Perifosine enhances bevacizumab-induced apoptosis and therapeutic efficacy by targeting PI3K/AKT pathway in a glioblastoma heterotopic model. Apoptosis 22: 1025-2034, 2017.

30. Zhao HF, Wang J, Shao W, Wu CP, Chen ZP, To ST and Li WP: Recent advances in the use of PI3K inhibitors for glioblastoma multiforme: Current preclinical and clinical development. Mol Cancer 16: 100, 2017. 